\title{
APPROXIMATION AND INTERPOLATION BY COMPLEX SPLINES ON THE TORUS
}

\author{
by T. N. T. GOODMAN, S. L. LEE and A. SHARMA
}

(Received 17th August 1987)

\section{Introduction}

Let $T^{2}=\left\{\left(e^{i x_{1}}, e^{i x_{2}}\right): 0 \leqq x_{j}<2 \pi, j=1,2\right\}$ be a two dimensional torus and $r, s, t$ and $k$ be positive integers with $k>r+s+t-2$. Our main object is to study the approximation and interpolation properties of a class of smooth functions whose restrictions to each triangle of a three direction mesh lie in the linear span of $\left\{z_{1}^{\mu} z_{2}^{v}: 0 \leqq \mu \leqq r-1,0 \leqq v \leqq s-1\right.$, or $0 \leqq \mu \leqq r-1, r+s-1 \leqq \mu+v \leqq r+s+t-2$, or $0 \leqq v \leqq s-1, r+s-1 \leqq \mu+v \leqq r+s+t-2\}$ where $\left(z_{1}, z_{2}\right) \in T^{2}$.

The one dimensional analogue is the class of uniform complex splines on the circle studied in [1], [5], [8], [10]. By analogy with [5], [8] and [10], the basic tool for our investigation will be a class of multivariate complex B-splines on the torus $M_{\Gamma}$ which is a complex version of polynomial box splines, a subject which has received much interest recently (see the survey paper [6] and the reference therein).

The complex B-spline on the torus is a periodic case of a general class of compactly supported functions, known as the exponential box splines, introduced recently by Amos Ron [9].

In Section 2 we define the complex B-spline $M_{\Gamma}$ on the $d$-dimensional torus and give a short proof of a basic relation for $M_{\Gamma}$. Section 3 deals with those properties of the linear combinations of translates of $M_{\Gamma}$ which will be useful in the sequel. The proofs of the results in Section 3 are just slight modifications of those in [6] and [9] for polynomial and exponential box splines. In Section 4, we study the complex B-splines on a three direction mesh on the torus and state an interpolation problem. Section 5 deals with finite double Fourier series which is the tool for our solution of the interpolation problem. The solution is given in Section 6. In Section 7, we construct the Bernstein-Schoenberg type approximation operators on the torus.

\section{Definition and elementary properties of complex B-splines on the torus}

Let $d$ be a positive integer and $\Gamma$ a multiset consisting of a finite number of elements of the form $\gamma=(e, \lambda)$, where $e \in \mathbb{Z}^{d} \backslash\{0\}$ and $\lambda \in \mathbb{Z}$. We define the sets $V_{\Gamma}:\{e:(e, \lambda) \in \Gamma\}$, $\Lambda_{\Gamma}:=\{\lambda:(e, \lambda) \in \Gamma\}$, and assume that $\left\langle V_{\Gamma}\right\rangle=\mathbb{R}^{d}$. We shall also use the same notation $V_{\Gamma}$ 
to represent the $d \times|\Gamma|$ matrix whose columns are the vectors of $\Gamma$, and $\Lambda_{\Gamma}$ to stand for the vectors in $\mathbb{Z}^{|\Gamma|}$ whose components are the corresponding $\lambda$ 's. We also define the set $J(\Gamma):=\left\{J \subset \Gamma: V_{J}\right.$ is a basis of $\left.\mathbb{R}^{d}\right\}$. To each $J \in J(\Gamma)$, there exists a unique $\theta_{J} \in \mathbb{R}^{d}$ such that $\theta_{J} V_{J}=\Lambda_{J}$.

Let $h=2 \pi / k$, where $k$ is a positive integer, such that $h e \in[-\pi, \pi]^{d}$ for $\gamma=(e, \lambda) \in \Gamma$. We shall assume throughout that

$$
\left|\operatorname{det} V_{J}\right|=1, \quad J \in J(\Gamma)
$$

and

$$
0<\left|\left(\lambda-\theta_{J} e\right) h\right|<2 \pi, \quad \text { for } \gamma=(e, \lambda) \in \Gamma \backslash J .
$$

Let $M_{\Gamma}$ be a function on $\mathbb{P}^{d}$ defined by

$$
M_{\Gamma}(x)=\left(\frac{1}{2 \pi}\right)^{d} \sum_{v \in \mathbf{Z}^{d}}\left(M_{\Gamma}\right)_{v}^{\wedge} \exp i v x, \quad x \in \mathbb{R}^{d}
$$

where

$$
\left(M_{\Gamma}\right)_{v}^{\Lambda}=\prod_{\nu \in \Gamma}\left\{\frac{\exp i(\lambda-v e) h-1}{\lambda-v e}\right\}, \quad v \in \mathbb{Z}^{d}
$$

where the factors in the product are taken to be ih if the denominators equal zero. We shall see later that the restrictions (2.1) and (2.2) will ensure that $M_{\Gamma}(x)$ is a piecewise polynomial in $z:=\left(\exp i x_{1}, \ldots, \exp i x_{d}\right) \in T^{d}$. We shall call $M_{\Gamma}$ the complex polynomial B-spline on the $d$-dimensional torus defined by $\Gamma$.

The Fourier coefficients (2.4) show that $M_{\mathrm{r}}$ is a convolution of periodic distributions $\mathscr{U}_{y}, \gamma=(\lambda, e) \in \Gamma$, on $\mathbb{P}^{d}$ defined by

$$
\mathscr{U}_{\gamma}(\phi)=i \int_{0}^{h} \exp (i \lambda t) \phi(e t) d t, \quad \phi \in \tilde{D}\left(\mathbb{R}^{d}\right)
$$

The distribution $\mathscr{U}_{y}$ is supported on the line segment $\bigcup_{v \in Z^{d}}(2 \pi \nu+[0, h e])$. Hence, the $\operatorname{supp} M_{\Gamma}$ is contained in $\bigcup_{v \in Z^{d}}\left(2 \pi v+\sum_{e \in V_{\Gamma}}[0, h e]\right)=\bigcup_{v \in Z^{d}}\left(2 \pi v+\left\{V_{\Gamma} t: t \in[0, h]^{|\Gamma|}\right\}\right)$.

A straightforward computation of the Fourier coefficients shows that for each $J \in J(\Gamma)$, $M_{J}$ is a periodic function given by

$$
M_{J}(x)=\left\{\begin{array}{c}
\exp i \theta_{J} x, x \in \bigcup_{v \in \mathbb{Z}^{d}}\left(2 \pi v+\left\{V_{J} t: t \in[0, h)^{d}\right\}\right) \\
0, \text { otherwise. }
\end{array}\right.
$$

Furthermore, if $\langle\Gamma \backslash\{\gamma\}\rangle=\mathbb{R}^{d}$, then 


$$
M_{\Gamma}(x)=i \int_{0}^{h} M_{\Gamma \backslash\{y\}}(x-t e) \exp (i \lambda t) d t, \quad x \in \mathbb{R}^{d}
$$

where $\gamma=(e, \lambda) \in \Gamma$.

Based on the Fourier coefficients (2.4) we define, for $\gamma=(e, \lambda) \in \Gamma$ two operators, the differential operator $D^{\gamma} f:=i\left(i \lambda-D_{e}\right) f$, and the difference operator $\nabla^{\gamma} f:=f-$ $\exp (i \lambda h) f(\cdot-h e)$, where $D_{e}$ denotes the directional derivative along $e$. Then a straightforward calculation, using (2.4), gives

Proposition 2.1. For $\gamma \in \Gamma$,

$$
D^{\gamma} M_{\Gamma}=\nabla^{\lambda} M_{\Gamma \backslash\{\gamma\}}
$$

and if $v=e /\|e\|^{2}$, then

$$
D_{e}\left(\exp (-i \lambda(v \cdot)) M_{\Gamma}\right)=i \exp (-i \lambda(v \cdot)) \nabla^{\lambda} M_{\Gamma \backslash(v)} .
$$

The following result is similar to that of Ron [9], but we give a short and direct proof.

Proposition 2.2. If conditions (2.1) and (2.2) are satisfied,

$$
M_{\Gamma}=\sum_{J \in J(\Gamma)} a_{\Gamma}(J) \nabla^{\Gamma \backslash J} M_{J}
$$

where

$$
a_{\Gamma}(J)=\prod_{\gamma \in \Gamma \backslash J}\left(\theta_{J} e-\lambda\right)^{-1}
$$

and

$$
\nabla^{\Gamma \backslash J}=\prod_{y \in \Gamma \backslash J} \nabla^{\gamma}
$$

Proof. To each $J \in J(\Gamma), \lambda-\theta_{J} e=0 \forall \gamma=(e, \lambda) \in J$, and by (2.2) $\lambda-\theta_{J} e \neq 0 \forall \gamma=$ $(e, \lambda) \in \Gamma \backslash J$. Using a partial fraction decomposition (see [3]) we have for $u \in \mathbb{R}^{d}$ for which $\lambda-u e \neq 0 \forall \gamma=(e, \lambda) \in \Gamma$,

$$
\frac{1}{\prod_{\gamma \in \Gamma}(\lambda-u e)}=\sum_{J \in J(\Gamma)} \frac{1}{\prod_{\gamma \in \Gamma \backslash J}\left(\lambda-\theta_{J} e\right)} \frac{1}{\prod_{\gamma \in J}(\lambda-u e)},
$$

where the products are over $\gamma=(e, \lambda)$. Multiplying equation (2.12) by $\prod_{\gamma \in \Gamma}$ $\{\exp i(\lambda-v e) h-1\}$, and taking the limit as $u \rightarrow v$ gives 


$$
\prod_{\nu \in \Gamma}\left\{\frac{\exp i(\lambda-v e) h-1}{\lambda-v e}\right\}=\sum_{J \in J(\Gamma)} a_{\Gamma}(J) \prod_{y \in \Gamma \backslash J}\{1-\exp i(\lambda-v e) h\} \prod_{\gamma \in J}\left\{\frac{\exp i(\lambda-v e) h-1}{\lambda-v e}\right\}
$$

where the quotient $(\exp i(\lambda-v e) h-1) /(\lambda-v e)$ is equal to ih if the denominator is zero.

Using (2.4) and the relation

$$
\left(\nabla^{\Gamma \backslash J} M_{\Gamma}\right)_{v}^{\wedge}=\prod_{\gamma \in \Gamma \backslash J}\{1-\exp i(\lambda-v e) h\}\left(M_{\Gamma}\right)_{v}^{\wedge}
$$

we obtain

$$
\left(M_{\Gamma}\right)_{v}^{\Lambda}=\sum_{J \in J(\Gamma)} a_{\Gamma}(J)\left(\nabla^{\Gamma \backslash J} M_{\Gamma}\right)_{v}^{\Lambda} \quad \forall v \in \mathbb{Z}^{d}
$$

from which $(2.10)$ follows.

Remark. From (2.6) and (2.10) we see that $M_{\Gamma}$ is a linear combination of functions of the form $\exp \left(i \theta_{J} x\right), J \in J(\Gamma)$, on each open set not crossed by the boundaries of the translates of $\operatorname{supp} M_{\mathrm{r}}, J \in J(\Gamma)$, along $j h, j \in \mathbb{Z}^{\mathrm{d}}$. We shall call such a maximal open set a $\Gamma$-cell. Since $\mid\left(\operatorname{det} V_{J} \mid=1, M_{\Gamma}(x)\right.$ equals a polynomial in $z:=\left(e^{i x_{1}}, \ldots, e^{i x_{d}}\right) \in T^{d}$ in each $\Gamma$-cell.

\section{Translates of complex box splines}

Let $\Gamma$ be as in Section 2 and assume that $\left\langle V_{\Gamma}\right\rangle=\mathbb{R}^{d}$. Let $k$ be a positive integer, $h:=2 \pi / k$ and $\mathbb{G}_{h}^{d}:=\left\{\alpha \in \mathbb{P}^{d}: \alpha_{i}=0, h, \ldots,(k-1) h, i=1,2, \ldots, d\right\}$. By $(2.2)$ we have for each $J \in J(\Gamma),\left|\lambda-\theta_{J} e\right|<k$ for all $\gamma=(e, \lambda) \in \Gamma \backslash J$.

Proposition 3.1. Suppose conditions (2.1.) and (2.2) are satisfied. For each $J \in J(\Gamma)$,

$$
\sum_{\alpha \in G_{\overparen{h}}} \exp \left(i \Lambda_{J} \alpha\right) M_{\Gamma}\left(x-V_{J} \alpha\right)=C_{\Gamma}(J) \exp \left(i \theta_{J} x\right)
$$

holds for $x \in \mathbb{R}^{d}$, where

$$
C_{\Gamma}(J)=\prod_{\gamma \in \Gamma \backslash J}\left\{\frac{\exp i\left(\lambda-\theta_{J} e\right) h-1}{\lambda-\theta_{J} e}\right\}
$$

and the void product is taken to be 1.

Proof. The proof is by induction on $|\Gamma|$ and is a straightforward modification of Theorem 5.1 of [9]. If $|\Gamma|=d$, then $J(\Gamma)=\{\Gamma\}$. With $J=\Gamma, C_{\Gamma}(J)=1$ and $M_{J}(x)$ is given by (2.6). Hence 


$$
M_{J}\left(x-V_{J} \alpha\right)=\left\{\begin{array}{cl}
\exp \left(i \theta_{J} x\right) \exp \left(-i \Lambda_{J} \alpha\right), & x \in \bigcup_{v \in \mathbb{Z}^{d}}\left(2 \pi v+\left\{V_{J}(t+\alpha): t \in[0, h)^{d}\right\}\right) \\
0 & , \text { otherwise. }
\end{array}\right.
$$

Thus (3.1) holds for $|\Gamma|=d$.

Suppose $|\Gamma|>d$. Then for $J \in J(\Gamma)$ and $\gamma=(e, \lambda) \in \Gamma \backslash J$, by the convolution formula (2.7),

$$
\begin{aligned}
\sum_{a \in \mathbf{G}_{h}^{f}} \exp \left(i \Lambda_{J} \alpha\right) M_{\mathrm{r}}\left(x-V_{J} \alpha\right) \\
=i \int_{0}^{h} \exp (i \lambda t) \sum_{\alpha \in \mathbf{G}_{h}} \exp \left(i \Lambda_{J} \alpha\right) M_{\Gamma \backslash\{y\}}\left(x-V_{J} \alpha-t e\right) d t \\
=i C_{\Gamma \backslash(\gamma)}(J) \int_{0}^{h} \exp (i \lambda t) \exp \left(i \theta_{J}(x-t e)\right) d t \\
=C_{\Gamma}(J) \exp \left(i \theta_{J} x\right) .
\end{aligned}
$$

Remark. The constants $C_{\Gamma}(J) \neq 0$ for all $J \in J(\Gamma)$ because of the assumption that $\left|\lambda-\theta_{J} e\right|<k \forall \gamma=(e, \lambda) \in \Gamma \backslash J$.

Next, we shall prove

Proposition 3.2. Let

$$
S:=\sum_{\alpha \in \mathrm{G}_{h}} a_{\alpha} M_{\Gamma}(\cdot-\alpha)
$$

Then for each $\gamma \in \Gamma$,

$$
D^{\gamma} S=\sum_{\alpha \in G_{h}^{f}} \nabla^{\gamma} a_{\alpha} M_{\Gamma \backslash\langle y\}}(\cdot-\alpha)
$$

where the equation is interpreted in the sense of distribution if $M_{\Gamma \backslash \gamma\}}$ is supported on a set of measure zero.

Proof. Suppose $\gamma=(e, \lambda) \in \Gamma$. By (2.8),

$$
\begin{aligned}
& D^{\gamma} S=\sum_{a \in \mathbf{G}_{\tilde{h}}} a_{a} \nabla^{\gamma} M_{\mathrm{r} \backslash\{y\}}(\cdot-\alpha) \\
& =\sum_{\alpha \in G \mathscr{h}} a_{\alpha} M_{\Gamma \backslash\{\gamma\}}(\cdot-\alpha)-\exp (i \gamma h) \sum_{\alpha \in G h} a_{\alpha} M_{\Gamma \backslash\{\gamma\}}(\cdot-\alpha-h e) \\
& =\sum_{\alpha \in G_{h}^{中}}\left\{a_{\alpha}-\exp (i \lambda h) a_{\alpha-h e}\right\} M_{\Gamma \backslash\lceil\rceil}(\cdot-\alpha)
\end{aligned}
$$


from which (3.3.) follows.

We shall now show that the conditions (2.1) and (2.2) imply that the translates $M_{\Gamma}(\cdot-\alpha), \alpha \in \mathbb{G}_{h}^{d}$ are locally linearly independent.

Proposition 3.3 Suppose the conditions (2.1) and (2.2) hold. Then the translates $M_{\Gamma}(\cdot-\alpha), \alpha \in \mathbb{G}_{h}^{d}$ are locally linearly independent.

Proof. The idea of the proof is the same as in [9]. If $A$ is any non-empty set in a $\Gamma$-cell, then by Propositions 2.2 and 3.1, the span $\left\{M_{\Gamma}(\cdot-\alpha) \mid \alpha \in \mathbb{G}_{h}^{d}\right\}$ is precisely span $\left\{\exp i \theta_{J} x: J \in J(\Gamma)\right\}$ and so has dimension $|J(\Gamma)|$. By a result of Dahmen and Micchelli ([4, Theorem 3.1]), the number of $\alpha$ 's for which $M_{\Gamma}(\cdot-\alpha)$ has support intersecting $A$ is less than or equal to $\sum_{J_{\in J}(\Gamma)}\left|\operatorname{det} V_{J}\right|=|J(\Gamma)|$. Hence the translates $M_{\Gamma}(x-\alpha) \neq 0$ for $x \in A$, $\alpha \in \mathbb{G}_{h}^{d}$ form a basis for the span of $\left\{\left.M_{\Gamma}(\cdot-\alpha)\right|_{A}: \alpha \in \mathbb{G}_{h}^{d}\right\}$.

Corollary. $\operatorname{supp} M_{\Gamma}=\bigcup_{v \in \mathbb{Z}^{d}}\left(2 \pi v+\left\{V_{\Gamma} t: t \in[0, h]^{|\Gamma|}\right\}\right)$.

Proof. This holds for $|\Gamma|=d$. By induction, using (2.9), $D_{e}\left(\exp (-i \lambda v x) M_{\Gamma}(x)\right) \neq 0$ on any $\Gamma$-cell in $\bigcup_{v \in \mathbb{Z}^{d}}\left(2 \pi v+\left\{V_{\Gamma} t: t \in[0, h]^{|\Gamma|}\right\}\right)$.

\section{Complex B-splines on a three direction mesh on the torus}

Let $k$ be a positive integer, $h:=2 \pi / k, \omega=\exp i h$ and let $e^{1}=(1,0), e^{2}=(0,1)$ and $e^{3}=(1,1)$. We consider the complex B-spline $M_{r s t}(z):=M_{\Gamma}(x), z=\left(z_{1}, z_{2}\right)=\left(e^{i x_{1}}, e^{i x_{2}}\right) \in T^{2}$, where $M_{\Gamma}$ is defined by $\Gamma=\left\{\left(e^{1}, 0\right), \ldots,\left(e^{1}, r-1\right), \quad\left(e^{2}, 0, \ldots,\left(e^{2}, s-1\right)\right.\right.$, $\left.\left(e^{3}, r+s-1\right), \ldots,\left(e^{3}, r+s+t-2\right)\right\}$, and $r, s$ and $t$ are positive integers. By (2.2), the Fourier coefficients of $M_{r s t}$ are given by

$$
\left(M_{r s t}\right)_{v}^{\Lambda}=a_{r v_{1}} b_{s v_{2}} c_{t v_{1} v_{2}}, \quad v=\left(v_{1}, v_{2}\right) \in \mathbb{Z}^{2}
$$

where

$$
\begin{aligned}
& a_{r v_{1}}=\prod_{j=0}^{r-1} \frac{\omega^{j-v_{1}}-1}{j-v_{1}}, \quad b_{s v_{2}}=\prod_{j=0}^{s-1} \frac{\omega^{j-v_{2}}-1}{j-v_{2}} \\
& c_{t v_{1} v_{2}}=\prod_{j=0}^{t-1} \frac{\omega^{r+s-1+j-v_{1}-v_{2}}-1}{r+s-1+j-v_{1}-v_{2}}, \quad \omega=\exp i h,
\end{aligned}
$$

and the factors in the product (4.2) are taken to be equal in when the denominators vanish.

Let $J(\Gamma):=J_{1}(\Gamma) \cup J_{2}(\Gamma) \cup J_{3}(\Gamma)$, where

$$
J_{1}(\Gamma):=\left\{\left\{\left(e^{1}, \mu\right),\left(e^{2}, v\right)\right\}: \mu=0, \ldots, r-1, v=0, \ldots, s-1\right\},
$$




$$
\begin{gathered}
J_{2}(\Gamma):=\left\{\left\{\left(e^{1}, \mu\right),\left(e^{3}, \rho\right)\right\}: \mu=0, \ldots, r-1, \rho=r+s-1, \ldots, r+s+t-2\right\}, \\
J_{3}(\Gamma):=\left\{\left\{\left(e^{2}, v\right),\left(e^{3}, \rho\right)\right\}: v=0, \ldots, s-1, \rho=r+s-1, \ldots, r+s+t-2\right\} . \\
\text { For } J \in J_{1}(\Gamma), \theta_{J}=(\mu, v), \mu=0, \ldots, r-1, v=0, \ldots, s-1, \text { for } \\
J \in J_{2}(\Gamma), \theta_{J}=(\mu, \rho-\mu), \mu=0, \ldots, r-1, \rho=r+s-1, \ldots, r+s+t-2, \text { and for } \\
J \in J_{3}(\Gamma), \theta_{J}=(\rho-v, v), v=0, \ldots, s-1, \rho=r+s-1, \ldots, r+s+t-2 .
\end{gathered}
$$

The $\left|\operatorname{det} V_{J}\right|=1$ for each $J \in J(\Gamma)$. Furthermore, for $J \in J(\Gamma)$ and $\gamma=(e, \lambda) \in \Gamma \backslash J, 0<$ $\left|\lambda-\theta_{J} e\right| \leqq r+s+t-2$. Therefore, condition (2.2) is satisfied if we assume that $r+s+t-$ $2<k$. This restriction and (4.2) implies that $M_{r}(x)$ is not a polynomial, but a proper spline function. The support,

$$
\operatorname{supp} M_{r s t}=\left\{\left(z, z_{2}\right)=\left(e^{i x_{1}}, e^{i x_{2}}\right): 0 \leqq x_{1} \leqq(r+t) h, 0 \leqq x_{2} \leqq(s+t) h,-s h \leqq x_{1}-x_{2} \leqq r h\right\}
$$

and by (2.6) and (2.10) we see that the restriction of $M_{r s t}$ to each "triangle" bounded by the mesh lines corrresponding to $x_{1}=j h, x_{2}=j h$ and $x_{1}+x_{2}=j h, j \in \mathbb{Z}$, lies in the span of

$$
\begin{gathered}
\left\{z_{1}^{\mu} z_{2}^{v}: 0 \leqq \mu \leqq r-1,0 \leqq v \leqq s-1, \quad \text { or } \quad 0 \leqq \mu \leqq r-1,\right. \\
r+s-1 \leqq \mu+v \leqq r+s+t-2, \quad \text { or } \quad 0 \leqq v \leqq s-1, \quad r+s-1 \leqq \mu+v \leqq r+s+t-2\} .
\end{gathered}
$$

Since conditions (2.1) and (2.2) are satisfied, the translates

$$
M_{r s t}\left(z_{1} \omega^{-a_{1}}, z_{2} \omega^{-\alpha_{2}}\right), \alpha=\left(\alpha_{1}, \alpha_{2}\right) \in K:=\left\{\left(v_{1}, v_{2}\right) \in \mathbb{Z}^{2}: 0 \leqq v_{i}<k, i=1,2\right\},
$$

are linearly independent. We shall consider the following interpolation problem.

Problem I: Given complex numbers $y_{\beta}, \beta \in K$, find $\left(c_{\alpha}\right)_{\alpha \in K}$ such that

$$
\sum_{\alpha \in K} c_{\alpha} M_{r s t}\left(\omega^{\beta_{1}-\alpha_{1}}, \omega^{\beta_{2}-\alpha_{2}}\right)=y_{\beta}, \quad \forall \beta \in K
$$

The corresponding problem of interpolation by polynomial box spline has been solved by de Boor, Hollig and Riemenschneider [2]. For the one dimensional case of uniform complex splines on the circle, the corresponding problem has been solved by Ahlberg, Nilson and Walsh [1] and Schoenberg [10]. Following Schoenberg, we shall formulate the Problem $I$ in terms of finite Fourier series. 


\section{Double finite Fourier series and the interpolation problem}

Take $k \geqq 2$, and set $h=2 \pi / k, \omega=\exp i h$. Consider an array $\left\{a_{\alpha}: \alpha \in \mathbb{Z}^{2}\right\}$ satisfying $a_{\alpha+\beta k}=a_{\alpha}$ for any $\beta \in \mathbb{Z}^{2}$. We define its Fourier coefficients $\left\{\hat{a}_{v}: v \in \mathbb{Z}^{2}\right\}$ by

$$
\hat{a}_{v}=\frac{1}{k^{2}} \sum_{\alpha \in K} a_{\alpha} \omega^{-v \alpha}
$$

Clearly, $\hat{a}_{v+\beta k}=\hat{a}_{v}$ for any $\beta \in \mathbb{Z}^{2}$, and

$$
a_{\alpha}=\sum_{v \in K} \hat{a}_{v} \omega^{\alpha v} .
$$

If we denote by $\Omega$ the $k^{2} \times k^{2}$ matrix $\left(\omega^{\alpha \beta}\right)_{\alpha, \beta \in K}$, then $\Omega^{-1}$ is the matrix $\left(1 / k^{2}\right)\left(\omega^{-\alpha \beta}\right)_{\alpha, \beta \in K}$, and the relations (5.1) and (5.2) can be written in matrix form

$$
\left(a_{\alpha}\right)_{\alpha \in K}=\Omega\left(\hat{a}_{v}\right)_{v \in K} .
$$

Furthermore, if $A$ denotes the $k^{2} \times k^{2}$ matrix $\left(a_{\alpha-\beta}\right)_{\alpha, \beta \in K}$, then $\Omega^{-1} A \Omega=k^{2} \operatorname{diag}\left(\hat{a}_{v}\right)_{v \in K}$. In particular

$$
A \text { is non singular if and only if } \hat{a}_{v} \neq 0 \forall v \in K \text {. }
$$

By (2.3) the complex B-spline $M_{r s t}$ on the two dimensional torus can be written as

$$
M_{r s t}(z)=\frac{1}{4 \pi^{2}} \sum_{v \in Z^{2}}\left(M_{r s t}\right)_{v}^{\Lambda} z_{1}^{v_{1}} z_{2}^{\nu_{2}}, \quad z=\left(z_{1}, z_{2}\right) \in U^{2}
$$

where $\left(M_{r s t}\right)_{v}^{\Lambda}$ is given by equation (4.1). For $\alpha \in K$

$$
M_{r s t}\left(\omega^{\alpha_{1}}, \omega^{\alpha_{2}}\right)=\frac{1}{4 \pi^{2}} \sum_{\nu \in \mathbf{Z}^{2}}\left(M_{r s t}\right)_{v}^{\wedge} \omega^{\alpha v}
$$

which can be written as

$$
M_{r s t}\left(\omega^{\alpha_{1}}, \omega^{\alpha_{2}}\right)=\frac{1}{4 \pi^{2}} \sum_{v \in K} A_{v} \omega^{\alpha \nu}
$$

where

$$
A_{v}=\sum_{\gamma \in \mathbf{Z}^{2}}\left(M_{r s t}\right)_{v+k \gamma}^{\Lambda}
$$

The interpolation problem (4.4) is uniquely solvable if and only if the matrix 


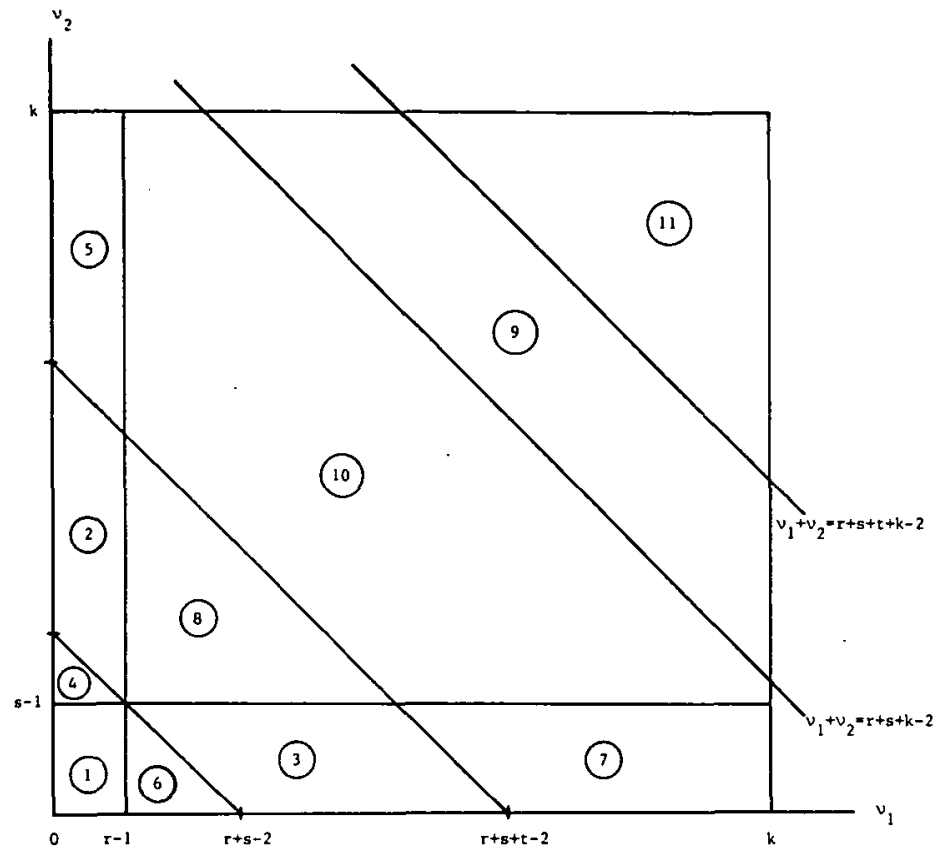

FIGURE $1 \quad K=\left\{\left(v_{1}, v_{i}\right): 0 \leqq v_{2} \leqq k-1, i=1,2\right\}$.

$\left(M_{r s t}\left(\omega^{\beta_{1}-a_{1}}, \omega^{\beta_{2}-\alpha_{2}}\right)\right)_{\alpha, \beta \in K}$ is non singular which is equivalent to $A_{v} \neq 0$ for all $v \in K$. By (4.1)

$$
A_{v}=\sum_{\gamma \in Z^{2}} a_{r v_{1}+k \gamma_{1}} b_{s v_{2}+k \gamma_{2}} c_{t v_{1}+k \gamma_{1} v_{2}+k \gamma_{2}}, \quad v \in K
$$

where

$$
\begin{gathered}
a_{r v_{1}+k \gamma_{1}}=\prod_{j=0}^{r-1} \frac{\omega^{j-v_{1}}-1}{j-v_{1}-k \gamma_{1}}, \\
b_{s v_{2}+k \gamma_{2}}=\prod_{j=0}^{s-1} \frac{\omega^{j-v_{2}}-1}{j-v_{2}-k \gamma_{2}}, \\
c_{t v_{1}+k \gamma_{1} v_{2}+k \gamma_{2}}=\prod_{j=0}^{t-1} \frac{\omega^{r+s-1+j-v_{1}-v_{2}}-1}{r+s-1+j-v_{1}-v_{2}-k \gamma_{1}-k \gamma_{2}} .
\end{gathered}
$$

\section{Solution of the interpolation problem}

In order to show that the finite Fourier coefficients $A_{v}$ are non zero, we shall partition $K$ into several regions (Figure 1 ) and look at the expressions for $A_{v}$ in each case. 
Case 1. $0 \leqq v_{1} \leqq r-1$ and $0 \leqq v_{2} \leqq s-1$. Then $a_{r v_{1}} \neq 0$ and $a_{r v_{1}+k \gamma_{1}}=0$ for $\gamma_{1} \neq 0$. Similarly $b_{s v_{2}} \neq 0$ and $b_{s v_{2}+k \gamma_{2}}=0$ for $\gamma_{2} \neq 0$. Thus

$$
A_{v}=\prod_{j=0}^{r-1}\left(\frac{\omega^{j-v_{1}}-1}{j-v_{1}}\right) \prod_{j=0}^{s-1}\left(\frac{\omega^{j-v_{2}}-1}{j-v_{2}}\right) \prod_{j=0}^{t-1}\left(\frac{\omega^{r+s-1+j-v_{1}-v_{2}}-1}{r+s-1+j-v_{1}-v_{2}}\right) .
$$

Case 2. $0 \leqq v_{1} \leqq r-1$ and $r+s-1 \leqq v_{1}+v_{2} \leqq r+s+t-2$. Then $a_{r v_{1}+k \gamma_{1}} \neq 0$ if and only if $\gamma_{1}=0$ and $c_{t v_{1}+k y_{1} v_{2}+k \gamma_{2}} \neq 0$ if and only if $\gamma_{1}+\gamma_{2}=0$. Then $A_{v}$ reduces to the expression in (6.1).

Case 3. $0 \leqq v_{2} \leqq s-1$ and $r+s-1 \leqq v_{1}+v_{2} \leqq r+s+t-2$. Then, as in Case 2, $A_{v}$ is as given in (6.1).

Case 4. $0 \leqq v_{1} \leqq r-1, r>1$ and $s \leqq v_{2} \leqq r+s-2-v_{1}$. Then $\prod_{j=0}^{s-1}\left(\omega^{j-v_{2}}-1\right) \neq 0$ and $\prod_{j=0}^{t-1}\left(\omega^{r+s-1+j-v_{1}-v_{2}}-1\right) \neq 0$, since $-r-s+2 \leqq j-v_{2} \leqq-1$, for $0 \leqq j \leqq s-1$ and $1 \leqq r+$ $s-1+j-v_{1}-v_{2}<k$, for $0 \leqq j \leqq t-1$. Hence

$$
A_{v}=B_{1} \sum_{\gamma=-\infty}^{\infty} \prod_{j=0}^{s-1} \frac{1}{j-v_{2}-k \gamma} \prod_{j=0}^{t-1} \frac{1}{r+s-1+j-v_{1}-v_{2}-k \gamma}
$$

where

$$
B_{1}=a_{r v_{1}} \prod_{j=0}^{s-1}\left(\omega^{j-v_{2}}-1\right) \prod_{j=0}^{t-1}\left(\omega^{r+s-1+j-v_{1}-v_{2}}-1\right) \neq 0 .
$$

Case 5. $0 \leqq v_{1} \leqq r-1$ and $r+s+t-1 \leqq v_{1}+v_{2} \leqq r+s+k-2$. Then $\prod_{j=0}^{s-1}\left(\omega^{j-v_{2}}-1\right) \neq 0$ and $\prod_{j=0}^{t-1}\left(\omega^{r+s-1+j-v_{1}-v_{2}}-1\right) \neq 0$, since $-k+1 \leqq j-v_{2} \leqq-t-1$ for $0 \leqq j \leqq s-1$ and $-k+1 \leqq r+s-1+j-v_{1}-v_{2} \leqq-1$, for $0 \leqq j \leqq t-1$. In this case $A_{v}$ reduces to the same expression as in (6.2).

Case 6. $0 \leqq v_{2} \leqq s-1, s>1$ and $r \leqq v_{1} \leqq r+s-2-v_{2}$.

Case 7. $0 \leqq v_{2} \leqq s-1$, and $r+s+t-1 \leqq v_{1}+v_{2} \leqq r+s+k-2$.

As in Cases 4 and 5, Cases 6 and 7 gives

$$
A_{v}=B_{2} \sum_{\gamma=-\infty}^{\infty} \prod_{j=0}^{r-1} \frac{1}{j-v_{1}-k \gamma} \prod_{j=0}^{t-1} \frac{1}{r+s-1+j-v_{1}-v_{2}-k \gamma}
$$

where 


$$
B_{2}=b_{s v_{2}} \prod_{j=0}^{r-1}\left(\omega^{j-v_{1}}-1\right) \prod_{j=0}^{t-1}\left(\omega^{r+s-1+j-v_{1}-v_{2}}-1\right) \neq 0
$$

Case 8. $r \leqq v_{1} \leqq k-1, s \leqq v_{2} \leqq k-1$ and $v_{1}+v_{2} \leqq r+s+t-2$. Then $\prod_{j=0}^{r-1}\left(\omega^{j-v_{1}}-1\right) \neq$ $0, \prod_{j=0}^{s-1}\left(\omega^{j-v_{2}}-1\right) \neq 0$ and $c_{t v_{1}+k \gamma_{1} v_{2}+k \gamma_{2}} \neq 0$ if and only if $\gamma_{1}+\gamma_{2}=0$. Hence

$$
A_{v}=B_{3} \sum_{\gamma=-\infty}^{\infty} \prod_{j=0}^{r-1} \frac{1}{j-v_{1}-k \gamma} \prod_{j=0}^{s-1} \frac{1}{j-v_{2}-k \gamma},
$$

where

$$
B_{3}=c_{t v_{1} v_{2}} \prod_{j=0}^{r-1}\left(\omega^{j-v_{1}}-1\right) \prod_{j=0}^{s-1}\left(\omega^{j-v_{2}}-1\right) \neq 0
$$

Case 9. $r+s+k-1 \leqq v_{1}+v_{2} \leqq r+s+t+k-2$. In this case $v_{1} \geqq r$ and $v_{2} \geqq s$. Furthermore $c_{t v_{1}+k \gamma_{1} v_{2}+k \gamma_{2}} \neq 0$ if and only if $\gamma_{1}+\gamma_{2}=-1$. Hence

$$
\begin{aligned}
A_{v} & =c_{t v_{1} v_{2}-k} \sum_{\gamma_{1}+\gamma_{2}=-1} \prod_{j=0}^{r-1}\left(\frac{\omega^{j-v_{1}}-1}{j-v_{1}-k \gamma_{1}}\right) \prod_{j=0}^{s-1}\left(\frac{\omega^{j-v_{2}}-1}{j-v_{2}-k \gamma_{2}}\right) \\
& =B_{4} \sum_{\gamma=-\infty}^{\infty} \prod_{j=0}^{r-1} \frac{1}{j-v_{1}-k \gamma} \prod_{j=0}^{s-1} \frac{1}{j-v_{2}+k \gamma+k},
\end{aligned}
$$

where

$$
B_{4}=c_{t v_{1} v_{2}-k} \prod_{j=0}^{r-1}\left(\omega^{j-v_{1}}-1\right) \prod_{j=0}^{s-1}\left(\omega^{j-v_{2}}-1\right) \neq 0
$$

Case 10. $r+s+t-1 \leqq v_{1}+v_{2} \leqq r+s+k-2$. In this case $v_{1} \geqq r$ and $v_{2} \geqq s$ which implies that $\prod_{j=0}^{r-1}\left(\omega^{j-v_{1}}-1\right) \neq 0$ and $\prod_{j=0}^{s-1}\left(\omega^{j-v_{2}}-1\right) \neq 0$. Furthermore, the above inequalities for $v_{1}+v_{2}$ implies that $-k+1 \leqq r+s-1+j-v_{1}-v_{2} \leqq-1$ for $j=0,2, \ldots, t$. Hence, we also have $\prod_{j=0}^{t-1}\left(\omega^{r+s-1+j-v_{1}-v_{2}}-1\right) \neq 0$, and therefore we can write

$$
A_{v}=B_{5} \sum_{\gamma \in Z^{2}} \prod_{j=0}^{r-1} \frac{1}{j-v_{1}-k \gamma_{1}} \prod_{j=0}^{s-1} \frac{1}{j-v_{2}-k \gamma_{2}} \prod_{j=0}^{t-1} \frac{1}{r+s-1+j-v_{1}-v_{2}-k \gamma_{1}-k \gamma_{2}}
$$

where

$$
B_{5}=\prod_{j=0}^{r-1}\left(\omega^{j-v_{1}}-1\right) \prod_{j=0}^{s-1}\left(\omega^{j-v_{2}}-1\right) \prod_{j=0}^{t-1}\left(\omega^{r+s-1+j-v_{1}-v_{2}}-1\right) \neq 0
$$


Case 11. $r+s+t+k-1 \leqq v_{1}+v_{2} \leqq 2 k-2$. In this case, a similar analysis shows that $A_{v}$ is also given by (6.6).

From the above expressions for $A_{v}$, it is clear that if $r, s$ and $t$ are all even, then $A_{v} \neq 0$ for $v \in K$. Thus, in this case, the interpolation is uniquely solvable. We state the above result as

Theorem 6.1. Given complex numbers $y_{\beta}, \beta \in K$, there exists a unique sequence $\left(c_{\alpha}\right)_{\alpha \in K}$ such that (4.4) holds if $r$, $s$ and $t$ are even.

Remark. Theorem 6.1 is the solution of a particular case of a more general interpolation problem which can be stated as follows:

Problem II. Given complex numbers $y_{\beta}, \beta \in K$, find $\left(c_{\alpha}\right)_{\alpha \in K}$ such that

$$
\sum_{\alpha \in K} c_{\alpha} M_{r s t}\left(\omega^{\beta_{1}-\alpha_{1}+\varepsilon_{1} / 2}, \omega^{\beta_{2}-\alpha_{2}+\varepsilon_{2} / 2}\right)=y_{\beta}, \quad \beta \in K,
$$

where $\varepsilon=\left(\varepsilon_{1}, \varepsilon_{2}\right), \varepsilon_{i} \in\{0,1\}, i=1,2$. Theorem 6.1 gives a solution to the case $\varepsilon=0$. We conjecture that Problem II is uniquely solvable if $\left(\varepsilon_{1}, \varepsilon_{2}\right)=(r+t, s+t) \bmod 2$.

\section{Bernstein-Schoenberg type operator}

We shall consider the complex B-splines $M_{r s t}(z), z \in T^{2}$. Since $\left|\operatorname{det} V_{J}\right|=1$ for all $J \in J(\Gamma)$, by Proposition 3.1, we have for each $J$

$$
C_{\mathrm{r}}(J)^{-1} \sum_{\alpha \in K} \omega^{\theta_{J} \alpha} M_{r s t}\left(z_{1} \omega^{-\alpha_{1}}, \omega^{-\alpha_{2}}\right)=z^{\theta_{J}}, \quad z=\left(z_{1}, z_{2}\right) \in T^{2},
$$

where

$$
C_{\Gamma}(J)=\prod_{\gamma \in \Gamma \backslash J}\left(\frac{\omega^{\lambda-\theta_{s e}}-1}{\lambda-\theta_{J} e}\right)
$$

and we have used the standard multivariate notation $z^{\theta_{J}}=z_{1}^{\mu} z_{2}^{v}, \theta_{J}=(\mu, v)$.

Let $J_{0}=\left\{\left(e^{1}, 0\right),\left(e^{2}, 0\right)\right\}$. Then $\theta_{J_{0}}=0$ and

$$
C_{\Gamma}\left(J_{0}\right)=\left(\begin{array}{c}
r+s-2 \\
r-1
\end{array}\right) \prod_{j=1}^{r-1}\left(\omega^{j}-1\right) \prod_{j=1}^{s-1}\left(\omega^{j}-1\right) \prod_{j=1}^{t-1}\left(\omega^{r+s+j+1}-1\right) /(r+s+t-2) !
$$

We now normalise the B-spline $M_{r s t}$ and set

$$
N_{r s t}=C_{\mathrm{r}}\left(J_{0}\right)^{-1} M_{r s t}
$$

It follows from (7.1) that 


$$
\sum_{\alpha \in K} N_{r s t}\left(z_{1} \omega^{-\alpha_{1}}, z_{2} \omega^{-\alpha_{2}}\right)=1 \forall z=\left(z_{1}, z_{2}\right) \in T^{2},
$$

and for each $J$.

$$
\left(C_{\Gamma}\left(J_{0}\right) / C_{\Gamma}(J)\right) \sum_{\alpha \in X} \omega^{\theta_{J} \alpha} N_{r s t}\left(z_{1} \omega^{-\alpha_{1}}, z_{2} w^{-\alpha_{2}}\right)=z^{\theta_{J}}, \quad z \in T^{2} .
$$

We shall show that there is a unique linear operator

$$
(S f)(z)=\sum_{\alpha \in \mathbf{K}} f\left(\tau_{\alpha}\right) N_{r s t}\left(z_{1} \omega^{-\alpha_{1}}, z_{2} \omega^{-\alpha_{2}}\right), \quad z \in T^{2},
$$

where $f$ is defined on a polyannulus, which reproduces $z^{\theta_{J_{1}}}$ and $z^{\theta_{J_{2}}}$ for any two distinct $J_{1}, J_{2}$ in $J(\Gamma)$. Let $\theta_{J_{1}}=\left(\mu_{1}, \mu_{2}\right)$ and $\theta_{J_{2}}=\left(\mu_{2}, v_{2}\right)$, where for $i=1,2,0 \leqq \mu_{i} \leqq r-1$, $0 \leqq v_{i} \leqq s-1$, or $0 \leqq \mu_{i} \leqq r-1, r+s-1 \leqq \mu_{i}+v_{i} \leqq r+s+t-2$, or $0 \leqq v_{i} \leqq s-1, r+s-1 \leqq \mu_{i}+$ $v_{i} \leqq r+s+t-2$. In view of (7.5) the above requirement give

$$
\tau_{\alpha}^{\theta J_{1}}=\frac{C_{\mathrm{\Gamma}}\left(J_{0}\right)}{C_{\Gamma}\left(J_{1}\right)} \omega^{\theta J_{1 \alpha}}, \quad \tau_{a}^{\theta j_{2}}=\frac{C_{\Gamma}\left(J_{0}\right)}{C_{\Gamma}\left(J_{2}\right)} \omega^{\theta J_{2 a}},
$$

which can be written as

$$
\begin{aligned}
& \tau_{\alpha 1}^{\mu_{1}} \tau_{\alpha 2}^{v_{1}}=\frac{C_{\Gamma}\left(J_{0}\right)}{C_{\Gamma}\left(J_{1}\right)} \omega^{\mu_{1} \alpha_{1}+v_{1} \alpha_{2}} \\
& \tau_{\alpha 1}^{\mu_{2}} \tau_{\alpha 2}^{v_{2}}=\frac{C_{\Gamma}\left(J_{0}\right)}{C_{\Gamma}\left(J_{2}\right)} \omega^{\mu_{2} \alpha_{1}+v_{2} \alpha_{2}}
\end{aligned}
$$

where $\tau_{\alpha}=\left(\tau_{\alpha 1}, \tau_{\alpha 2}\right)$ and $\alpha=\left(\alpha_{1}, \alpha_{2}\right) \in K$. A straightforward computation gives

$$
\begin{aligned}
& \tau_{\alpha 1}=C_{\Gamma}\left(J_{0}\right)^{\left(v_{2}-v_{1}\right) \Delta^{-1}}\left(C_{\Gamma}\left(J_{2}\right)^{v_{1}} / C_{\Gamma}\left(J_{1}\right)^{v_{2}}\right) \Delta^{-1} \omega^{\alpha_{1}} \\
& \tau_{\alpha 2}=C_{\Gamma}\left(J_{0}\right)^{\left(\mu_{1}-\mu_{2}\right) \Delta^{-1}}\left(C_{\Gamma}\left(J_{1}\right)^{\mu_{2}} / C_{\Gamma}\left(J_{2}\right)^{\mu_{1}}\right) \Delta^{-1} \omega^{\alpha_{2}}
\end{aligned}
$$

where $\Delta=\mu_{1} v_{2}-\mu_{2} v_{1}$. Thus the operator (7.6) with $\tau_{\alpha}=\left(\tau_{\alpha 1}, \tau_{\alpha 2}\right)$ defined by (7.9) reproduces the constant function and the functions $z^{\theta J_{1}}$ and $z^{\theta J_{2}}$, where $\theta_{J_{1}}=\left(\mu_{1}, v_{1}\right)$, $\theta_{J_{2}}=\left(\mu_{2}, v_{2}\right)$.

For simplicity, we shall assume that $J_{1}=\left\{\left(e^{1}, 1\right),\left(e^{2}, 0\right)\right\}$ and $J_{2}=\left\{\left(e^{1}, 0\right),\left(e^{2}, 1\right)\right\}$. Then $\theta_{J_{1}}=(1,0), \theta_{J_{2}}=(0,1)$, and $z^{\theta_{J_{1}}}=z_{1}$ and $z^{\theta_{J_{2}}}=z_{2}$. By (7.9).

$$
\tau_{\alpha}=\left(\frac{C_{\Gamma}\left(J_{0}\right)}{C_{\Gamma}\left(J_{1}\right)} \omega^{\alpha_{1}}, \frac{C_{\Gamma}\left(J_{0}\right)}{C_{\mathrm{r}}\left(J_{2}\right)} \omega^{\alpha_{2}}\right)
$$

and it follows from (7.2) by elementary computation that 


$$
\tau_{\alpha}=\left(R_{1} \omega^{\alpha_{1}+(r+t) / 2}, R_{2} \omega^{\alpha_{2}+(s+t) / 2}\right),
$$

where

$$
\begin{aligned}
& R_{1}=\left(\frac{\sin (r-1) \pi / k}{(r-1) \pi / k}\right)\left(\frac{\sin (r+s+t-2) \pi / k}{(r+s+t-2) \pi / k}\right) /\left(\frac{\sin \pi / k}{\pi / k}\right)\left(\frac{\sin (r+s-2) \pi / k}{(r+s-2) \pi / k}\right) \\
& R_{2}=\left(\frac{\sin (s-1) \pi / k}{(s-1) \pi / k}\right)\left(\frac{\sin (r+s+t-2) \pi / k}{(r+s+t-2) \pi / k}\right) /\left(\frac{\sin \pi / k}{\pi / k}\right)\left(\frac{\sin (r+s-2) \pi / k}{(r+s-2) \pi / k}\right) .
\end{aligned}
$$

It is easy to see that

$$
1-R_{i}=0\left(k^{-2}\right)
$$

We shall prove the following.

Theorem 7.1 Let $f(z)$ be defined and continuous on a polyannulus $A^{2}:=\left\{\left(z_{1}, z_{2}\right): \rho_{i} \leqq\right.$ $\left.\left|z_{i}\right| \leqq 1, i=1,2\right\}$, where $0<\rho_{i}<R_{i}$. Then the operator $S f$ defined by (7.6), where $\tau_{\alpha}$ is given by (7.11), reproduces the functions $1, z_{1} z_{2}$, and

$$
|(S f)(z)-f(z)| \leqq M \omega\left(f ; k^{-1}\right), z \in T^{2},
$$

where $M$ is independent of $f$ and $k$, and $\omega\left(f ; k^{-1}\right)$ denotes the modulus of continuity.

We shall first establish a simple lemma.

Lemma 1. For fixed integers $r, s, t$,

$$
\left|N_{r s t}(z)\right|=0(1), \quad z \in T^{2} .
$$

Proof. First, we observe that $\left|M_{110}(z)\right|=0(1)$, and using (2.7) by induction on $n=r+s+t$, it is easy to see that $\left|M_{r s t}(z)\right|=0\left(k^{-n+2}\right)$. The result then follows from (7.3), since

$$
\begin{aligned}
\left|C_{\Gamma}\left(J_{0}\right)\right|= & \left(\begin{array}{c}
r+s-2 \\
r-1
\end{array}\right) \prod_{j=1}^{r-1}(2 \sin j \pi / k) \prod_{j=1}^{s-1}(2 \sin j \pi / k) \\
& \prod_{j=0}^{t-1}(2 \sin (r+s+j-1) \pi / k) /(r+s+t-2) ! \\
= & 0\left(k^{-n+2}\right) .
\end{aligned}
$$

Proof of Theorem 7.1. By (7.4) and (7.6), we have 


$$
(S f)(z)-f(z)=\sum_{\alpha \in K}\left(f\left(\tau_{\alpha}\right)-f(z)\right) N_{r s t}\left(z_{1} \omega^{-\alpha_{1}}, z_{2} \omega^{-\alpha_{2}}\right), \quad z \in T^{2}
$$

For a fixed $\left(z_{1}, z_{2}\right)=\left(e^{i x_{1}}, e^{i x_{2}}\right) \in T^{2}, N_{r s t}\left(z_{1} \omega^{-\alpha_{1}}, z_{2} \omega^{-\alpha_{2}}\right) \neq 0$ if and only if $\alpha_{1} h<x_{1}<$ $\left(r+t+\alpha_{1}\right) h, \alpha_{2} h<x_{2}<\left(s+t+\alpha_{2}\right) h$, and $\left(-s+\alpha_{1}-\alpha_{2}\right) h<x_{1}-x_{2}<\left(r+\alpha_{1}-\alpha_{2}\right) h$. Therefore from (7.11), in view of (7.13), a straightforward computation gives

$$
\left|\tau_{\alpha}-z\right|:=\left(\left|\tau_{\alpha 1}-z_{1}\right|^{2}+\left|\tau_{\alpha 2}-z_{2}\right|^{2}\right)^{1 / 2}=0\left(k^{-1}\right)
$$

The result now follows from (7.15) and (7.16).

Remarks. 1. The order of approximation in (7.14) is best possible for the class of continuous functions on $A^{2}$. For if $f\left(z_{1}, z_{2}\right)=\left|z_{1}\right|^{1 / 2}$, then there exists a constant $C$ such that $|(S f)(z)-f(z)| \geqq C k^{-1}$.

2. The Bernstein-Schoenberg operator (7.6), with $\tau_{x}$ given by (7.11), reproduces the constant function and the functions $z_{1}$ and $z_{2}$. In this case the function $f$ is defined on the polyannulus $A^{2}$. However, for any $f \in C\left(T^{2}\right)$, we can define a linear operator

$$
(S f)(z)=\sum_{\alpha \in K} f\left(\omega^{\alpha_{1}+(r+t) / 2}, \omega^{\alpha_{2}+(s+t) / 2}\right) N_{r s t}\left(z_{1} \omega^{-\alpha_{1}}, z_{2} \omega^{-\alpha_{2}}\right), \quad z \in T^{2}
$$

This operator $\tilde{S} f$ does not reproduce $z_{1}, z_{2}$, and as in the proof of Theorem 7.1 , we have

Theorem 7.2. For $f \in C\left(T^{2}\right)$,

$$
|(\tilde{S} f)(z)-f(z)| \leqq M_{1} \omega\left(f, k^{-1}\right), \quad z \in T^{2},
$$

where $M_{1}$ is independent of $f$ and $k$.

\section{REFERENCES}

1. J. H. Ahlberg, E. N. Nilson and J. L. Walsh, Properties of analytic splines I: Complex polynomial splines, J. Analysis and Appl. 33 (1971), 234-257.

2. C. DE Boor, K. Hollig and S. D. Riemenschneider, Bivariate cardinal interpolation by splines on a three direction mesh, Illinois J. Math. 29 (1985), 555-566.

3. W. Dahmen and C. A. Micchelli, On limits of multivariate B-splines, J. Analyse Math. 39 (1981), 256-278.

4. W. Dahmen and C. A. Micchelli, On the local linear independence of translates of a box spline, Stud. Math. Appl. 82 (1985), 243-263.

5. T. N. T. Goodman and S. L. LeE, B-splines on the circle and trigonometric B-splines, Proc. Conference on Approx. Theory, St. John's, Newfoundland (Reidel Pub. Co., 1983), 297-325.

6. K. Hollıc, Box splines, Approximation Theory V (Academic Press, Boston, Mass., 1986), 71-95.

7. R. Q. J IA, On the linear independence of translates of box splines, J. Approx. Theory 40 (1984), 158-160. 
8. C. A. Micchelli and A. Sharma, Spline functions on the circle: Cardinal L-splines revisited, Canad. J. Math. 32 (1980), 1459-1473.

9. Amos Ron, Exponential box splines, to appear in Constructive Approximation.

10. I. J. Schoenberg, On polynomial spline functions on the circle I and II, Proc. Conference on Constructive Theory of Functions, Budapest (1972), 403-433.

Department of Mathematical

and Computing SCIEnCes

UNIVERSITY OF DUNDEE

DUNDEE DDI 4HN

SCOTLAND

Department of Mathematics

UNIVERSITY OF AlberTA

EDMONTON

Alberta T6G 2G1

Canada
School of Mathematical and Computer Sciences SCience University of Malaysia Penang 11800

Malaysia AND

Department of Mathematics National University of Singapore

10 Kent Crescent SiNGAPORE 0511 